\title{
Radioactively Powered Electromagnetic Counterparts of Neutron Star Mergers
}

\section{B. D. Metzger ${ }^{* \dagger}$}

Department of Astrophysical Sciences, Princeton University; Princeton, NJ 08544

E-mail: bmetzger@astro.princeton.edu

\section{G. Martínez-Pinedo, A. Arcones}

GSI Helmholtzzentrum für Schwerionenforschung, Planckstr. 1, D-64291 Darmstadt, Germany

\section{S. Darbha, E. Quataert, D. Kasen}

Astronomy Department and Theoretical Astrophysics Center; University of California; Berkeley, CA 94720

\section{R. Thomas, P. Nugent}

Lawrence Berkeley National Laboratory; Berkeley, CA, 94720

\section{V. Panov}

Department of Physics, University of Basel, Klingelbergstr. 82, CH-4056; Basel, Switzerland

\section{N. T. Zinner}

Department of Physics, Harvard University, Cambridge, MA 02138, USA

The most promising astrophysical sources of gravitational waves (GWs) for ground-based interferometers such as LIGO and Virgo are the inspiral and merger of binary neutron star (NS) and black hole systems. However, maximizing the scientific benefits of a GW detection will require identifying a coincident electromagnetic counterpart. One of the most likely sources of isotropic emission from NS mergers is a supernova-like transient powered by the radioactive decay of $r$ process elements synthesized in the merger ejecta. We present the first calculations of the optical transients from NS mergers that self consistently determine the radioactive heating using a nuclear reaction network and which determine the resulting light curve with a Monte Carlo radiation transfer calculation. Due to the rapid evolution and low luminosity of NS merger transients, optical counterpart searches triggered by a GW detection will require close collaboration between the GW and astronomical communities. NS merger transients may also be detectable following a short duration Gamma-Ray Burst or blindly with present or upcoming optical transient surveys.

11th Symposium on Nuclei in the Cosmos

19-23 July 2010

Heidelberg, Germany.

\footnotetext{
* Speaker.

${ }^{\dagger}$ NASA Einstein Fellow
} 
The inspiral and merger of neutron star (NS) and black hole binaries are among the most promising sources of gravitational waves (GWs) for ground-based interferometers such as LIGO and Virgo. Although a direct GW detection would signal a monumental breakthrough for fundamental physics, maximizing the resulting science will require identifying a coincident electromagnetic (EM) counterpart [1]. This is important for several reasons, including (1) identifying the host galaxy and environs of the merger; (2) allowing for a direct and independent measurement of the Hubble constant; (3) enhancing the effective sensitivy of present GW detectors by improving the confidence level of a given event.

A commonly discussed EM signal associated with NS-NS/NS-BH mergers is a short-duration Gamma-Ray Burst (GRB). However, the association between short GRBs and NS mergers is not yet definitive [2] and because GRB jets are relativistically beamed, only a small fraction of events (those with jets pointed towards Earth) are detectable [3]. The advent of large-scale optical surveys with increasing sensitivity, rapid cadence, and sky-area coverage is revolutionizing the study of transient objects (e.g. Palomar Transient Factory [PTF]; Pan-STARRs; Large Synoptic Survey Telescope [LSST]). Given these present and anticipated future capabilities, the most promising EM counterpart is arguably an isotropic, optical wavelength signal.

As first proposed by [4], a promising source of quasi-isotropic emission from NS mergers is a supernova-like transient powered by the radioactive decay of merger ejecta. Unlike Type Ia $\mathrm{SNe}$, which are powered by the decay of ${ }^{56} \mathrm{Ni}$ and ${ }^{56} \mathrm{Co}$, NS merger transients are powered by the decay of much heavier elements (mass number $A \gtrsim 130$ ), which are formed via rapid neutron capture ( $r$-process) nucleosynthesis following the ejecta's decompression from nuclear densities (e.g. $[5,6])$. Although the $r$-process itself lasts at most a few seconds, these newly-synthesized elements undergo fission and beta decays on much longer timescales as they descend to $\beta$-stability. The resulting energy powers detectable thermal emission once the ejecta expands sufficiently that photons may escape.

Neutron-rich material may be ejected both dynamically during the merger itself, or by (nuclear recombination-powered) winds from the accretion disk at later times (e.g. [9]). Typical values of the ejecta mass and speed are $M_{\mathrm{ej}} \approx 10^{-2} M_{\odot}$ and $v \approx 0.1 \mathrm{c}$, although $M_{\mathrm{ej}}$ may vary between events by more than order of magnitude. The EM luminosity peaks when the timescale for photon diffusion becomes less than the expansion timescale [7], which occurs at $t \approx t_{\text {peak }} \approx$ 0.5 days $\left(\frac{v}{0.1 c}\right)^{-1 / 2}\left(\frac{M_{\mathrm{ej}}}{10^{-2} M_{\odot}}\right)^{1 / 2}$. Due to the lower mass and faster speed of the ejecta from NS mergers relative to normal supernovae, emission peaks on a shorter timescale $\lesssim 1$ day, making these events a challenge to detect. The peak luminosity is approximately

$$
L_{\text {peak }} \approx \frac{\left.E_{\text {th }}\right|_{\text {peak }}}{t_{\text {peak }}} \approx 5 \times 10^{41} \operatorname{ergs~s}^{-1}\left(\frac{f}{10^{-6}}\right)\left(\frac{v}{0.1 c}\right)^{1 / 2}\left(\frac{M_{\mathrm{ej}}}{10^{-2} M_{\odot}}\right)^{1 / 2},
$$

where $\left.E_{\text {therm }}\right|_{t_{\text {peak }}} \equiv f M_{\mathrm{ej}} c^{2}$ is the thermal energy of the ejecta at $t \approx t_{\text {peak }}$, which we parameterize in terms of a dimensionless constant $f$. The detectability of NS mergers clearly depends sensitively on $f$, which must be determined self-consistently from the radioactive heating. In a recent work [10] we present the first calculations of the transient emission from NS mergers that uses a nuclear physics reaction network to calculate the radioactive heating of the ejecta $(\$ 1)$ and more accurately models the light curve and color evolution of the resulting EM transient using a radiative transfer code (§2). We briefly summarize this work below. 


\section{Nuclear Reaction Network and Radioactive Heating}

We employ a dynamical $r$-process network that includes neutron captures, photodissociations, $\beta$-decays and fission reactions (see $[10,11]$ for more details). Ejecta trajectories are from the numerical NS-NS merger calculations of [12]. Our results for the total radioactive heating rate $\dot{E}$ as a function of time following the ejection are shown in Figure 1. The large heating rate at early times is due to the $r$-process, which ends when neutrons are exhausted at $t \sim 1 \mathrm{~s} \sim 10^{-5}$ days. Heating on longer timescales results from the synthesized isotopes decaying back to stability.

Although the fast nuclei and electrons produced in the decays thermalize efficiently with the plasma via coloumb collisions, a small fraction of the $\beta$-decay energy is lost to neutrinos and $\gamma$-rays. We estimate that the total thermalization efficiency ranges between $\varepsilon_{\text {therm }} \approx 25 \%$ and unity, such that the effective value of $f$ defined in equation $(1)$ is $\approx 3 \times 10^{-6}$. Our results shown in Figure 1 are for one ejecta composition, but we have performed similar calculations considering different values of the electron fraction $Y_{e}$ and a different nuclear mass model. In all cases the radioactive heating on timescales $\approx 1$ days agrees with our fiducial model to within a factor of a few, suggesting that our results are relatively robust.

\section{Light Curves and Detection Prospects}

We calculate the light curves of NS merger ejecta using the time-dependent radiative transfer code SEDONA [8], which is modified to include the radioactive heating $\dot{Q}=\varepsilon_{\text {therm }} \dot{E}$ as shown in Figure 1. Although the total opacity of the ejecta is probably dominated by densely-packed UV lines, the available spectral line data for $r$-process elements is limited. We crudely account for the $r$-process element opacities by using the bound-bound lines of Fe, an assumption which may be justified because the third $r$-process peak elements $(A \sim 195)$ are also largely transition metals. Fortunately, the overall lightcurve shape, peak luminosity, and characteristic timescales are robust in spite of these uncertainties.

Figure 2 shows our results for the bolometric light curve, calculated for the model shown in Figure 1. The predicted peak luminosity is $\sim$ few $\times 10^{41} \mathrm{ergs} \mathrm{s}^{-1}$ (visual magnitude $M_{\mathrm{V}} \approx-15$ ), or a factor $\sim 10^{3}$ larger than the Eddington luminosity for a solar mass object. We therefore dub these events "kilo-novae," since standard novae are approximately Eddington-limited events.

A transient peaking at $L_{\text {peak }} \sim 3 \times 10^{41} \mathrm{erg} \mathrm{s}^{-1}$ (Fig. 2) corresponds to an apparent magnitude $V \sim 22-24$ at the edge of the Advance LIGO volume (distance $\sim 300$ and $\sim 650 \mathrm{Mpc}$ for NSNS/NS-BH mergers, respectively). Although this represents a realistic depth for a large telescope, the positional uncertainty of LIGO/Virgo detections will range from many arcminutes to degrees, such that a telescope with both sensitivity and a large field of view (e.g. LSST) is optimal for GW follow-up. Given the short duration $\sim 1$ day, rapid GW data reduction and dissemination of candidate detections to the astronomical community will be crucial. Encouragingly, recent efforts have begun to set up a GW data analysis pipeline to produce location-probability sky maps within $\sim 5-10$ minutes following a LIGO/Virgo detection [13].

Given the possible association between short GRBs and NS mergers, another method to search for kilonovae is with deep optical/IR observations on $\sim 1$ day timescale following the burst. Using the upper limits from refs. $[14,15]$ we constrain the ejecta mass to be $M_{\text {ej }} \lesssim 0.1 M_{\odot}$ and $\lesssim 10^{-3} M_{\odot}$ for GRBs 070724A and 050509b, respectively. The most promising candidate kilonova detection occured following GRB 080503, which showed an unusual rise in its optical afterglow at $t \sim 1$ day 


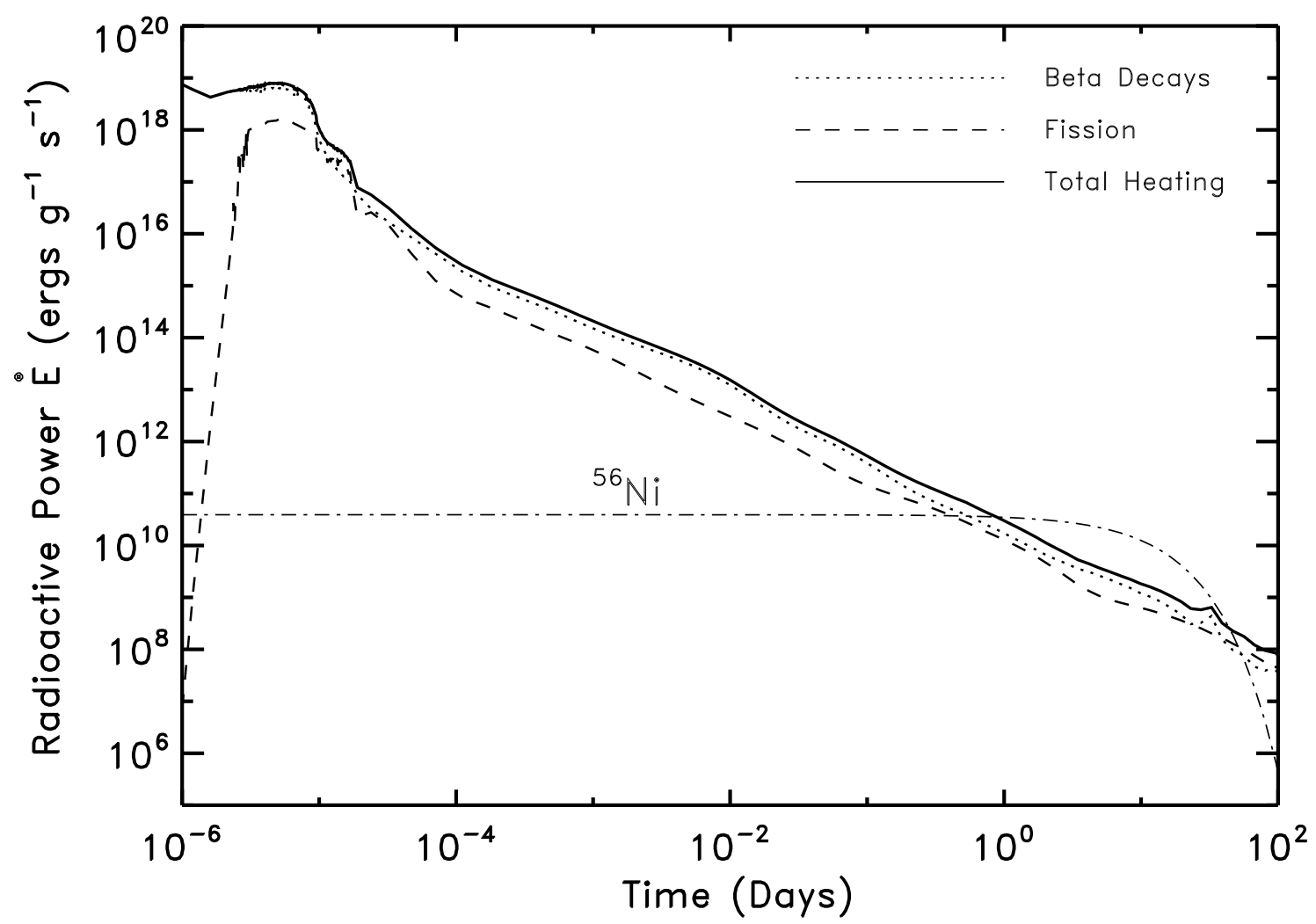

Figure 1: Radioactive heating rate per unit mass $\dot{E}$ in NS merger ejecta due to the decay of $r$-process material, calculated for NS merger ejecta with electron fraction $Y_{e}=0.1$ (see [10] for details). The total heating rate is shown with a solid line and is divided into contributions from $\beta$-decays (dotted line) and fission (dashed line). For comparison we also show the heating rate per unit mass produced by the decay chain ${ }^{56} \mathrm{Ni} \rightarrow{ }^{56} \mathrm{Co} \rightarrow{ }^{56} \mathrm{Fe}$ (dot-dashed line). On the timescales of interest for powering EM emission ( $t_{\text {peak }} \sim$ hours-days), most of the fission results from the spontaneous fission of nuclei with $A \sim 230-280$ and the $\beta$-decays of nuclei such as ${ }^{132,135} \mathrm{I},{ }^{128,129} \mathrm{Sb}$ and ${ }^{129} \mathrm{Te}$.

before rapidly fading over the next several days in a manner consistent with predictions [16]. The lack of a candidate host galaxy at the predicted redshift $z \approx 0.1$ and the coincident detection of bright X-ray emission, however, called into question a kilonova interpretation.

A final way to detect NS mergers is 'blindly' with present and upcoming optical transient surveys. Assuming an optimistic merger rate of $\sim 10^{-4} \mathrm{yr}^{-1}$ galaxy $^{-1}$, PTF(LSST) should detect up to $\sim 1\left(10^{3}\right)$ mergers per year, respectively. Obviously a major challenge is to uniquely identify merger transients sufficiently early to obtain follow-up photometry and spectra. A "smoking gun" feature of kilonovae would be the presence of optical absorption lines due to heavy neutron-rich elements (as in $r$-process enriched halo stars), although Doppler smearing due to the fast ejecta speed could lead to a rather featureless spectra.

\section{References}

[1] Bloom J. S., et al., 2009a, ArXiv e-prints: 0902.1527

[2] Berger, E. 2010, arXiv:1005.1068

[3] Rhoads J. E., 1999, ApJ, 525, 737 


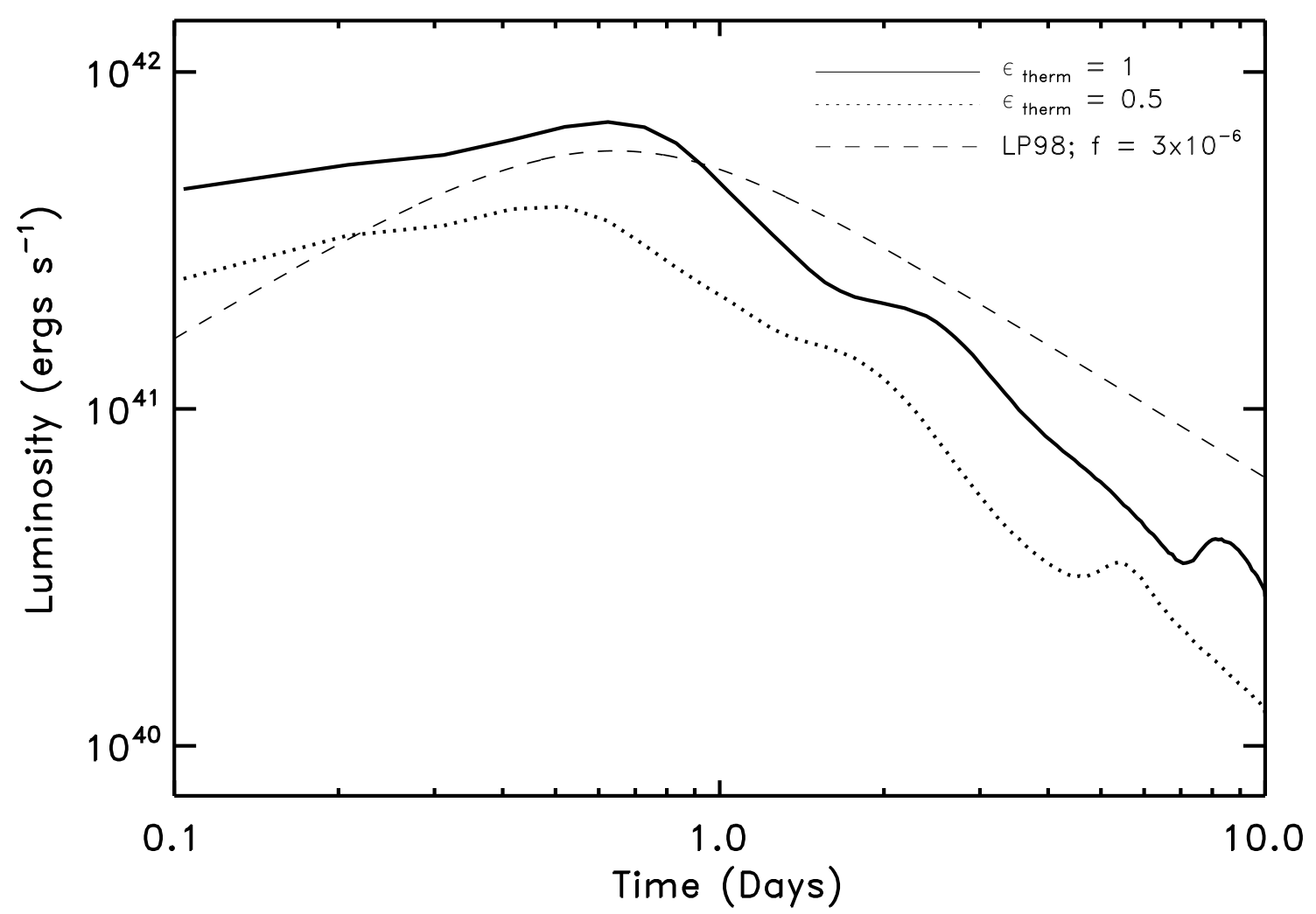

Figure 2: Bolometric light curve of the radioactively-powered transients from NS-NS/NS-BH mergers, calculated assuming a total ejecta mass $M_{\mathrm{tot}}=10^{-2} M_{\odot}$ with electron fraction $Y_{e}=0.1$ and mean outflow speed $v \simeq 0.1 \mathrm{c}$, and for two values of the thermalization efficiency, $\varepsilon_{\text {therm }}=1$ (solid line) and $\varepsilon_{\text {therm }}=0.5$ (dotted line), which roughly bracket our uncertainty. Also shown for comparison (dashed line) is a one-zone calculation as implemented in e.g. [4], assuming $f=3 \times 10^{-6}$ and the same values for $M_{\text {tot }}$ and $v$.

[4] Li L., Paczyński B., 1998, ApJ, 507, L59

[5] Lattimer J. M., Schramm D. N., 1974, ApJL, 192, L145

[6] Freiburghaus C., Rosswog S., Thielemann F., 1999, ApJL, 525, L121

[7] Arnett W. D., 1982, ApJ, 253, 785

[8] Kasen D., Thomas R. C., Nugent P., 2006, ApJ, 651, 366

[9] Metzger, B. D., Piro, A. L., \& Quataert, E. 2009, MNRAS, 396, 304

[10] Metzger, B. D., et al. 2010, MNRAS, 406, 2650

[11] Petermann, I., et al. 2008, Nuclei in the Cosmos (NIC X),

[12] Rosswog S., Liebendörfer M., Thielemann F., Davies M. B., Benz W., Piran T., 1999, AAP, 341, 499

[13] Kanner J., et al. 2008, Classical and Quantum Gravity, 25, 184034

[14] Hjorth J., et al., 2005, Nature, 437, 859

[15] Kocevski, D., et al. 2010, MNRAS, 404, 963

[16] Perley D. A., et al., 2009, ApJ, 696, 1871 